$57 \mid 2016$

Innovations pédagogiques dans l'enseignement des langues étrangères : perspective historique (XVle-XXle siècles)

\title{
Les pionniers du FLE en Espagne. De Sotomayor (1565) à Jaron (1688)
}

Antonio Gaspar Galán and J. Fidel Corcuera Manso

\section{(2) OpenEdition \\ Journals}

\section{Electronic version}

URL: https://journals.openedition.org/dhfles/4379

DOI: $10.4000 /$ dhfles.4379

ISSN: 2221-4038

\section{Publisher}

Société Internationale pour l'Histoire du Français Langue Étrangère ou Seconde

Printed version

Date of publication: 1 December 2016

Number of pages: $57-76$

ISSN: 0992-7654

\section{Electronic reference}

Antonio Gaspar Galán and J. Fidel Corcuera Manso, "Les pionniers du FLE en Espagne. De Sotomayor (1565) à Jaron (1688)", Documents pour I'histoire du français langue étrangère ou seconde [Online], 57 | 2016, Online since 01 February 2018, connection on 26 March 2023. URL: http:// journals.openedition.org/dhfles/4379; DOl: https://doi.org/10.4000/dhfles.4379

This text was automatically generated on 26 March 2023.

All rights reserved 


\title{
Les pionniers du FLE en Espagne. De Sotomayor (1565) à Jaron (1688)
}

\author{
Antonio Gaspar Galán and J. Fidel Corcuera Manso
}

\section{Introduction}

1 À l'époque de la Renaissance les nouvelles puissances européennes ont considéré que la langue était partie prenante $\mathrm{du}$ pouvoir politique. Conséquemment, l'affaire linguistique a été placée au centre des agendas royaux. La monarchie française a suivi les conseils d'écrivains, grammairiens et diplomates et a mis en œuvre une vraie politique linguistique. En effet, le «langage maternel françoys» reçoit à l'époque l'appui législatif de Charles VIII, Louis XII et surtout de François $\mathrm{I}^{\mathrm{er}}$ et il devient la langue nationale ${ }^{1}$. Les Ordonnances de Moulin, les Dispositions du mois de juin 1510, les Ordonnances d'Is-sur-Tille de 1535 et de Villers-Cotterêts de 1539, entre autres ${ }^{2}$, sont la base juridique sur laquelle ce « langage françoys » devient la langue française.

Pour l'Espagne de la Reconquête, de la Contre-Réforme et de l'Inquisition, il était tout à fait évident qu'un pouvoir unique s'accordait parfaitement à une religion unique et à une langue unitaire. Le castillan s'impose sur l'arabe, mais aussi sur le galicien, sur l'aragonais, sur le catalan... La réalité nationale et impériale a joué son rôle tout naturellement. Depuis le XIII ${ }^{\text {e }}$ siècle, le castillan était la langue officielle de la Chancellerie Royale à la suite d'un décret du roi Fernando III. Son fils Alfonso X Le Sage, parrain de l'École de Traducteurs de Tolède, fait de la langue de cette ville le modèle à suivre pour la rédaction des documents publics ${ }^{3}$.

La préface de la Gramatica sobre la lengua castellana de Nebrija (1492) affirme que la langue a toujours été "compañera del Imperio» (le latin et l'empire romain en constituent un très bel exemple pour Nebrija) et que les « pueblos barbaros \& naciones de peregrinas » soumis au pouvoir royal doivent « recebir las leies quel vencedor pone al vencido, \& con ellas nuestra lengua ", ce qui fait du castillan une partie essentielle de la politique royale. 


\section{Le contexte espagnol}

4 Nebrija avait indiqué la politique à suivre : la considération du castillan comme une langue comparable au latin est une idée que l'on peut repérer dans les textes de l'époque. Villalón (1997 : 36) déclare en 1550 à propos du Scholastico : « no es escripta la presente obra en latín, sino en nuestra castellana lengua, porque más façilmente dize el hombre lo que quiera en su propia lengua que en la peregrina ». Et il compare le castillan au latin et même au grec et à l'hébreu: «allende que la lengua que Dios y naturaleza nos ha dado no nos deue ser menos apazible ni menos estimada que la latina, griega y hebrea ». Quelques années plus tard, il publie à Anvers sa Gramática Castellana (1558), pour que tout le monde puisse apprendre le castillan et suivre l'exemple de l'empereur :

Forçome por el consiguiente a esta empresa ver el comun de todas las gentes inclinadas a esta dichosa lengua; y que les aplaze mucho y se preçian de hablar en ella. El Flamenco, el Italiano, Ingles, Frances. Y avn en Alemania se huelgan de la hablar: avnque se presume que sea alguna parte de causa ver que el nuestro Emperador Carlos se preçia de Español natural.

5 Aldrete (1606) considère que sa langue «No es inferior a cualquiera de las más celebradas pues en muchas virtudes I partes se les auentaja» et il présente au roi Philipe III des arguments touchant langue, culture et politique : «Estas i otras cosas tocantes à los Reinos de V. Magestad refiero en este libro, que segun creo, son de prouecho en la politica, de gusto en el conocimiento, de importancia para la lengua Castellana ». Et Correas utilise des arguments territoriaux pour montrer la supériorité du castillan : « su estenzión es sin comparazión más qe la Latina, porqe fué i es comun nuestra Castellana Española a toda España, qe es mayor más de un terzio qe Italia. Y hase estendido sumamente en estos 120 años por aqellas mui grandes provinzias del Nuevo-mundo de las Indias Ozidentales i Orientales» (Correas, $1626: 316)$. L'euphorie règne parmi les grammairiens, qui transfèrent au domaine linguistique le succès politique de l'Empire et qui considèrent que la langue castillane doit devenir la langue de ce nouvel Empire.

\section{La situation linguistique}

6 Des grammaires à l'usage des étrangers sont imprimées un peu partout en Europe pour satisfaire aux nouveaux besoins de communication. Pourtant le panorama est loin d'être homogène: il existe une différence considérable entre le grand nombre de grammaires publiées aux $\mathrm{XVI}^{e}$ et $\mathrm{XVII}^{\mathrm{e}}$ siècles en Europe pour apprendre le castillan et le peu de manuels parus en Espagne pour apprendre le français. Suárez Gómez l'avait déjà signalé en 1956 et justifiait cette différence par l'attitude volontairement "ignorante» des Espagnols de l'époque (2008: 148). Cela surprend aussi Alatorre (1998: 82) et aussi Lope Blanch (1986:53), qui se faisait l'écho de cette disproportion et concluait que «los españoles, al menos los del Siglo de Oro, no parecían estar muy interesados en aprender idiomas extranjeros $»^{4}$. Salazar $(1623: 70)$ insiste sur cette idée :

Les François de leur naturel sont curieux (...) ce que ie trouve le contraire en Espaignol (...) pource qu'il se trouvera dans Paris le tiers des Courtisans qui sçavent parler Castillan, et la plus part sans avoir esté en Espagne, dont ie me suis estonné 
que de tous ceux qui vindrent avec de Duc de Pastrane à Paris, il n’y en avoit pas six qui sçeussent parler François.

7 Morel-Fatio (1901: 86) remarque le rôle capital du castillan dans la Cour espagnole et les historiens Kamen (1997 : 232) et Vaca de Osma (1999 : 191) confirment la nécessité de traduire tous les documents. La liste du personnel de la Cour de Philippe II montre l'existence de trois traducteurs officiels, Juan Bret, Lucas Finger et Roberto Mofet, ce qui paraît étayer cette hypothèse (Martínez Millan, 2005 : Apéndice).

8 À notre avis, trois motifs peuvent expliquer cette attitude : la rivalité franco-espagnole à cause des guerres franco-espagnoles, la prépondérance culturelle de l'Empire et la politique linguistique qui prône le castillan comme langue de la diplomatie européenne.

9 Les relations entre la France et l'Empire se développent dans une ambiance belliqueuse. Les conflits territoriaux et religieux sont à l'ordre du jour et les deux puissances se disputent la suprématie européenne, ce qui ne favorise pas les échanges culturels. Ils sont aussi suffisamment connus les mots que le Roi Henri IV adresse à son fils ("Je ne veulx pas qu'il saiche seulement qu'il y ayt une Espagne $\left.{ }^{5} »\right)$, ou l'argument de César Oudin (1619) qui écrit sa Grammaire espagnole «afin qu'en lisant les Histoires de la conquiste des Indes, on voye les cruautez que les Espagnols y ont exercees ».

D'autre part, l'empire espagnol jouissait d'un considérable prestige culturel. Arredondo (1984) présente un panorama de traductions d'œuvres espagnoles pour expliquer le « extraordinario interés que despertaba todo lo español al otro lado de los Pirineos ». La prépondérance internationale du point de vue politique et économique s'accompagne d'une diffusion de la culture espagnole ${ }^{6}$.

11 Finalement, il y a un fond de politique linguistique que l'on peut repérer dans les attitudes, les textes et les discours ${ }^{7}$. Les écrits de Arias Montano sur la politique linguistique à suivre aux Pays-Bas (Lanoye : 2002), le discours prononcé en castillan par l'Empereur Charles Quint en avril 1536 à Rome devant le Pape Paulo III en présence des ambassadeurs européens (Alatorre, 1998 : 76), de même que son allocution en castillan devant le Senat génois (Viñaza, 1983 : XXIV et 1021) constituent des exemples de la volonté de diffusion du castillan sur le plan international.

Pour compléter ce panorama linguistique, il faut souligner que des nécessités impérieuses avaient attiré l'intérêt des Espagnols vers d'autres langues non européennes. D'un côté, la langue arabe et les autres langues péninsulaires: peu d'années après la parution de la Gramatica de Nebrija, les presses de Joan Rosembach publient le Vocabolari molt profitós per apendre lo catalan-alamany y lo alamany-catalan à Perpignan en 1502, et Pedro de Alcalá fait sortir à Granada son Vocabulista arábigo en 1505. De l'autre, la découverte du Nouveau Monde avait mis les Espagnols en contact avec toute une série de langues amérindiennes qu'il fallait comprendre pour des raisons politiques, économiques et/ou religieuses. Cette exigence communicative est à l'origine de beaucoup de manuels, dont le Vocabulario de la lengua castellana y mexicana de Alonso de Molina, par exemple, paru en 1540, un quart de siècle avant la grammaire de Sotomayor ${ }^{8}$.

13 Or, on peut conclure que le marché espagnol pour les manuels de français était vraiment restreint à l'époque, les seuls intéressés étant des nobles qui voulaient apprendre par "curiosité » ou qui devaient exercer des missions diplomatiques. Le centre mondial du commerce de l'époque, les Pays-Bas, fournissait des manuels 
plurilingues pour qui en avait besoin en raison de ses activités économiques et, en fait, l'activité éditoriale des dix-sept provinces sera l'origine intellectuelle des premiers manuels de français publiés en Espagne.

\section{Les manuels de français en Espagne}

\subsection{Les œuvres} qui ont joué le rôle de pionniers dans le contexte si peu propice de l'Espagne Impériale?

Pour la période qui fait l'objet de notre analyse, il n'y a que 7 manuels de français (dont 5 grammaires et 2 dictionnaires) parus en Espagne, pour apprendre le français à un public hispanophone. Bien entendu, certaines grammaires ont été parfois rééditées en Espagne ou constituent des rééditions/adaptations de manuels publiés à l'étranger ${ }^{9}$ :

-1565. Balthasar de Sotomayor. Grammatica con reglas muy prouechosas y necessarias para aprender a leer y escriuir la lengua Francesa, conferida con la Castellana, con un vocabulario copioso delas mesmas lenguas, Alcalá de Henares, Francisco de Cormellas y Pedro de Robles. ${ }^{10}$

17 -1565. Jacques de Liaño/Ledel. Vocabulario de los vocablos que mas comunmente se suelen usar, puestos por orden del Abecedario en Frances, y su declaracion en Español. Alcalá de Henares, Francisco de Cormellas y Pedro de Robles. ${ }^{11}$

18 -1635. Diego de Cisneros/de la Encarnación. Arte de grammatica francesa en Español, composé de trois livres. Madrid. ${ }^{12}$

19 -1642. Pere Lacavalleria. Dictionario Castellano, Dictionaire François, Dictionari Català (titre de la couverture) o Dictionari de tres llenguas, Castellana, Francesa y Catalana (titre de la licence) ${ }^{13}$.

20 -1647. Antonio Lacavalleria, Grammatica con reglas muy provechosas, y necessarias para aprender a ller, y escribir la lengua Francesa, conferida con la Castellana. Con un estilo de escrivir, hablar, y pronunciar las dos lenguas. Barcelona ${ }^{14}$.

21 -1673. Pedro Pablo Billet ${ }^{15}$. Gramatica francesa; dividida en dos partes, Saragoça. Billet signale qu'une première édition de ce travail avait vu le jour quelques mois auparavant ${ }^{16}$. Il a été conservé par contre plusieurs exemplaires des éditions postérieures (Madrid, 1688 et 1708 et Anvers, trois impressions sans date). L'édition de 1688 nous a permis de connaître la rivalité Billet-Jaron, deux maîtres de langue française qui possèdent deux puissants protecteurs à la Cour espagnole ${ }^{17}$.

-1688. Juan Pedro Jaron, Arte nuevamente compuesto de la Lengua Francesa por la Española, segun la nueva correción de Richelet à Madrid, à l'imprimerie de Lucas Antonio Bedmar y Baldivia. Ce manuel est dédié en français et en espagnol à « $\mathrm{N}$. Señora del Monte Carmelo", et a été approuvé par "don Francisco Cruzado, y Aragón ", trésorier de la reine, qui est à son tour le père de D. Esteban Cruzado, qui avait approuvé la grammaire madrilène de Billet.

23 Il faut citer deux références spéciales pour compléter cette relation de manuels de français pour les Espagnols : 
24 - a) Compendio util y necesario sobre las advertencias para la Pronunciacion de la lengua Francesa de Francisco Columbanu, écrit vers 1700, et cité par Palau (réf. 57343), mais aucun exemplaire n'est conservé actuellement.

- b) Balthasar Perez del Castillo, Arte, Grammatica y manera de bien hablar, screbir y leer la lengua Francesa. Le manuscrit inédit, rédigé avant la fin du XVI ${ }^{\mathrm{e}}$ siècle, est partiellement conservé à la Real Biblioteca de Madrid et compte environ une vingtaine de pages consacrées à la prononciation du français, les articles, le nom et le pronom ${ }^{18}$.

\section{2. Structure et sources des manuels}

Le Vocabulario de Liaño est composé d'un glossaire de 1495 mots (Corcuera et Gaspar, 1999 : XLVIII), d'un «Estilo para bien leer y hablar la lengua Francesa », d'un «Stile de bien escrire \& prononcer la langue Espaignole » et d'une série de textes en français et en espagnol suivant le modèle des Manières de langage. Ses sources ont été déterminées depuis longtemps par Bourland (1933), Roldán (1977) et Lépinette (1990). Il paraît évident que Liaño a utilisé la Vtil, y breve institvtion, para aprender los principios $y$ fundamentos de la lengua Hespañola de 1555 et le Vocabulario de Quatro lingue de 1558 de Berlaimont.

La grammaire de Sotomayor a dû être connue à l'époque, puisqu'elle apparaît très tôt dans les répertoires espagnols (Antonio, 1696 : t. I, 186). Nombre d'auteurs ont signalé les ressemblances de la Grammatica de Sotomayor et des Coniugaisons de G. Meurier de 1558 , à tel point que, pour certains, il s'agirait plutôt d'une copie littérale. Il est vrai que l'auteur de Tolède suit de près les Coniugaisons, mais son rôle dépasse celui d'un simple copiste et ses sources ne sont pas si évidentes qu'on le croyait. Nous avons signalé dans l'étude qui précède notre édition critique (Gaspar et Corcuera, 2015), que Sotomayor a effectivement utilisé cette source, mais que les différences entre les deux textes sont remarquables. Plus d'un quart de la grammaire de Sotomayor (26 fol.) n'a rien à voir avec les Coniugaisons de 1558. Pourtant les coïncidences entre sa grammaire et la nouvelle édition des Coniugaisons parue en 1568 sont presque totales. Or, l'œuvre de Sotomayor avait été publié en 1565 , ce qui nous amène à poser une triple hypothèse. La première, il a existé une nouvelle édition remaniée des Coniugaisons entre 1558 et 1564 ; la seconde, une édition des Berlaimont a incorporé et complété les Coniugaisons avant 1564 ; finalement, Sotomayor a rédigé sa grammaire à partir de la Grammaire Françoise de 1557 et des Coniugaisons de 1558 de Meurier et, à son tour, son œuvre a été utilisée par les Coniugaisons de 1568.

Pour la première possibilité, il faut signaler que Niederehe (1994) ne cite pas de nouvelles éditions des Coniugaisons entre 1558 et 1564. Plus récemment, Pettegree et Wlasby (2011) ont affirmé qu'il existe une édition de 1564 des Coniugaisons faite à Anvers par Waesberghe et conservée sous la côte VadSlg JA4560 dans les fonds de la Kantonsbibliothek St. Gallen en Suisse. Cependant, cette référence comprend cinq ouvrages de Meurier: Dialogues (1562), Deviz familiers (1564), Propos puerils (1565), La guirlande des ieunes filles (1564) et un exemplaire des Coniugaisons, qui n'est pas de 1564, mais de 1565.

29 L'hypothèse d'une édition des Berlaimont incorporant les Coniugaisons modifiées avant la parution de la grammaire de Sotomayor s'avère improbable. Il existe huit impressions avec des titres différents (Dictionnaire/Vocabulaire/Colloquia) entre 1558 et 
1565, mais aucune n'inclut les Coniugaisons. Les exemplaires conservés montrent que la première édition à reprendre les Coniugaisons de Meurier est celle de 1568.

Ce qui nous amène à conclure que soit il a existé une édition hybride des Berlaimont/ Meurier antérieure à 1565, inconnue jusqu'à présent, soit Sotomayor a rédigé une combinaison des matériaux verbes/vocabulaires provenant de Meurier et de Berlaimont et que son travail a été utilisé pour les Coniugaisons de $1568^{19}$.

Diego de Cisneros constitue un cas rare dans le panorama des manuels espagnols de français de l'époque. Sa grammaire respecte le modèle classique de la tradition latine : il divise son œuvre en trois parties dont la première traite des "lettres et leur prononciation ", la deuxième "Les éléments du discours ", c'est-à-dire article, nom, pronom, verbe, participe, adverbe (incluant l'interjection), préposition, conjonction; et la troisième "la construction des parties de l'oraison ». Son objectif est une explication rationnelle de la grammaire française à partir de principes plutôt philosophiques qui continuent les présupposés de Sanctius et anticipent en quelque sorte les grammaires rationalistes (Yllera, 1983, Lépinette, 1996 et Martínez Gavilán, 2002). Pour ce qui est de ses sources, Bruña (2000: 75) voit dans cette rationalité le reflet de son modèle: «Cisneros distribue rationnellement la matière de son traité [...]. Cette distribution équilibrée est due sans doute à la fidélité de l'auteur à l'égard du De Francicae linguae recta pronunciatione (1584), auquel il emprunte non seulement l'organisation générale de la matière, mais aussi l'ordre des commentaires à l'intérieur de chaque chapitre ».

Billet organise sa grammaire suivant le modèle classique hérité du latin. L'édition de Zaragoza (première édition espagnole de son œuvre) se compose de deux parties et d'un art poétique «Compendio breue de la Poesia Francesa ». La première partie comprend « los primeros rudimentos », le traitement des lettres et leur prononciation, et se termine par la description des parties du discours (article, nom, pronom, préposition, interjections et conjonctions, adverbes et verbes). La deuxième partie est consacrée à la syntaxe (« De la oración, o la construcción en general »), et se termine par un «Paralelo Castellano, y Francés/Paralelle François, \& Espagnol », qui constitue un recueil de tours de langue spécifiques du français et de l'espagnol. Billet utilise un procédé contrastif produit de sa large expérience de professeur de français en Espagne et déclare dans son introduction au «Paralelle de l'éloquence » qu'il a utilisé et corrigé le dictionnaire d'Oudin. Pourtant, Bruña (2000: 81$)$ considère que sa source principale n'est pas Oudin, mais « la grammaire de Maupas (1607), à partir de laquelle Billet a construit un canevas où sont venus s'insérer aussi bien ses apports personnels que ceux provenant d'autres auteurs ». Le résultat est une grammaire descriptive qui inaugure la série de " grammaires des observations » (Lépinette, 1996 : 159) publiées en Espagne au XVIII ${ }^{\mathrm{e}}$ siècle.

La grammaire de Jaron n'a pas de table des matières, mais l'avertissement au lecteur déclare que le manuel aborde « los Elementos de la pronunciación, con toda claridad, de los Nombres, y sus declinaciones, de los Pronombres, de las quatro Conjugaciones, y de la explicacion de los Verbos, assi Regulares, como Irregulares, ò Anomalos ». Cette organisation de la grammaire suit le «Latin, para mayor inteligencia de los Eruditos, conformandome en todo lo possible con la Lengua Castellana ».

34 Pour la première fois, un auteur de grammaire française en Espagne avoue d'emblée sa source d'inspiration et renvoie à son modèle pour les passages difficiles, ce qui montrerait qu'il y a vers la fin du XVII ${ }^{\mathrm{e}}$ siècle une exigence normative de prestige qui n'existait pas auparavant dans les manuels espagnols de français. Jaron annonce dès le 
titre le nom de Richelet comme le modèle qu'il a suivi pour rédiger son Arte: Arte nuevamente compuesto de la lengua Francesa por la Española, según la nueva Corrección de Richelet. L'auteur du Dictionnaire françois de 1680 est non seulement source d'inspiration de l'œuvre et autorité pour les éventuels doutes, mais protection contre les critiques : « No faltarà quien murmure desta Obra ; pero para abrigarme de la Censura, confiesso, que todo este Opusculo lo hè escogido del Vocabulario de Richelet, Autor el mas Moderno de quantos han escrito, y el que ha puesto la lengua francesa en su ultima perfección ».

\subsection{Les auteurs}

Une perspective sur les auteurs de ces manuels, que nous pouvons classer en quatre groupes, nous permet de compléter le panorama espagnol du FLE à l'époque.

- a. Maîtres de langues de la noblesse, professeurs de français de personnages importants qui ont enseigné pendant un certain temps de manière plus ou moins professionnalisée, et qui ont rédigé une grammaire à l'aide des notes des cours qu'ils ont fait pendant des années. C'est le cas de Billet et de Jaron. Les deux sont français d'origine, le premier bourguignon, le second parisien). Pour eux, les manuels constituent une pièce fondamentale de leur travail quotidien comme professeurs particuliers. De sorte qu'ils se confient à des auteurs prestigieux, citent leurs sources d'inspiration et font référence à des académiciens pour montrer que leur français est dans la norme. Billet est appelé « el Nebrija de la Francesa Gramática » par son disciple Francisco de Barrio. Jaron, de sa part, se réfugie sous l'autorité de Richelet.

37 - b. Grammairiens amateurs agissant sous commande, qui possèdent des connaissances de français et d'espagnol, qui fréquentent la Cour ou des personnages de la noblesse, et qui rédigent un manuel « s'inspirant » des grammaires précédentes. C'est le cas de Liaño et Sotomayor. Liaño est un « moço de capilla » de la reine Isabelle de Valois qui rédige son Vocabulaire à partir des Berlaimont. Sotomayor, un voisin de Tolède dont on ne connaît pas le métier. La rédaction du vocabulaire et de la grammaire ainsi que la publication en un seul volume ont bien pu être le résultat d'une initiative pour le compte des imprimeurs, de sorte que Pedro de Robles et Francisco de Cormellas ont peut-être joué un rôle essentiel dans cette affaire. Lorenzo de Robles, fils de Pedro de Robles, est à son tour l'auteur d'un petit manuel d'espagnol adressé aux Français qui est une copie presque littérale de la grammaire de César Oudin, ce qui contribuerait à confirmer cette hypothèse.

38 - c. Membres d'ordres religieux qui possèdent des connaissances de français et de grammaire. Diego de la Encarnación se présente comme «carme déchaussé » professeur de théologie. Il publie deux fois sa Grammaire, la première à Douai et la deuxième à Madrid. À l'époque de la publication de la grammaire de Madrid il se présente comme Diego de Cisneros, ce qui ne pose pas de problèmes d'identification. Cisneros est aussi le traducteur du premier livre des Essais de Montaigne, manuscrit intitulé Experiencias y varios discursos de Miguel señor de Montaña et conservé à la BN de Madrid (MSS/5635), dont la publication a été interdite par l'Inquisition.

- d. Imprimeurs sans formation spécifique de langues, qui vont remanier des manuels antérieurs pour avoir des avantages économiques. Ainsi, Antonio Lacavalleria profite de l'appartenance de la Catalogne à la couronne française en 1647, pour refaire et 
réimprimer le vocabulaire de Ledel et la grammaire de Sotomayor en s'appropriant littéralement du travail sans en citer les auteurs.

\section{Pour conclure} considération de la langue française comme une langue de prestige. Le français est surtout la langue des ennemis, et la monarchie espagnole met sur pied une politique linguistique nationale et internationale qui préconise la prépondérance du castillan. L'arabe et les langues amérindiennes attirent très tôt l'attention des grammairiens pour des raisons évidentes : la première grammaire de l'arabe paraît en 1505, et dès 1540 il existe des manuels des langues des territoires d'outremer. Le succès politique s'accompagne du prestige culturel et le castillan est présent dans les manuels spécifiques, bilingues et plurilingues, publiés en Europe depuis le début du XVI ${ }^{e}$. En Espagne, la Gramática de Nebrija de 1492 considère d'une telle importance l'apprentissage du castillan par les étrangers qu'elle inclut un livre $\mathrm{V}$ pour ceux qui désirent apprendre le castillan (« De las introduciones de la lengua castellana para los que de estraña lengua querrán deprender»). Pour ce qui est du français, il faudra attendre jusqu'en 1565 pour voir la parution de la première grammaire publiée en Espagne, celle de Baltasar de Sotomayor.

À différence de ce qui arrive dans d'autres pays (Du Wez, Cauchie, Garnier, Pillot, Meurier, Berlaimont, etc.), les grammaires de français publiées en Espagne sont adressées à un public qui ne connaît pas le latin. En effet, aucun des manuels espagnols de français n'est rédigé en latin et, même si le modèle utilisée est plurilingue, l'adaptation espagnole en simplifie le contenu, les manuels étant adressés de manière presque générale " au curieux lecteur ». Les besoins culturels ou économiques du pays n'exigent pas d'autres types de publications. Les Pays-Bas fournissaient suffisamment de grammaires pour apprendre d'autres langues à partir du castillan, de l'anglais, de l'allemand, de l'italien ou du latin pour les commerçants ou les diplomates désireux d'apprendre le français.

En fait, cette région européenne se révèle un espace stratégique pour l'enseignement du français en Espagne, étant donné que les premiers manuels ne sont pas originels, mais sont des grammaires inspirées (souvent de très près) d'œuvres précédentes. Les manuels qui ont servi de modèle pour les premières grammaires proviennent des presses d'Anvers ou de Louvain, et Cisneros publie la première édition de sa grammaire à Douai. Ce n'est qu'à partir de la moitié du XVII ${ }^{e}$ siècle que les auteurs s'inspirent de modèles français.

Finalement, les auteurs constituent aussi une spécificité du contexte espagnol. Tandis qu'en Angleterre, aux Pays-Bas, en France ou en Allemagne, les auteurs de manuels sont des professionnels de l'enseignement des langues (professeurs de la noblesse ou d'établissements d'enseignement), en Espagne ce sont des amateurs, grammairiens vocationnels ou imprimeurs qui cherchent un bénéfice économique: Liaño est un adjudant de chapelle de la Cour, Sotomayor un voisin de Tolède dont on ne connait pas le métier, Lacavallería un imprimeur qui adapte des manuels antérieurs, etc. En 1635, 
un religieux publie à Madrid la deuxième édition d'une grammaire d'inspiration philosophique qu'il avait déjà publiée aux Pays-Bas. Ce n'est qu'en 1673 qu'un professeur de français rédige en Espagne un manuel de français.

\section{BIBLIOGRAPHY}

ALATORRE, Antonio (1998) [1996]. El apogeo del castellano, Madrid : Fondo de Cultura Económica. ALDRETE, Bernardo (1606). Del origen y principio de la lengua castellana o romance que oi se usa en España. Roma : Carlo Wllieto.

ANÓNIMO (1555) (1977). Vtil, y breve institvtion, para aprender los principios y fundamentos de la lengua Hespañola. Institution tres brieue \& tres utile, pour aprendre les premiers fondemens, de la langue Españole. Institutio breuissima \& vtilissima ad discenda prima rudimenta linguae Hispanicae. Lovanii, ex officina Bartholomei Gravii. Éd. fac-similée et étude de A. Roldán, Madrid : C.S.I.C.

ANTONIO, Nicolás (1672). Bibliotheca Hispana Nova. Roma, N. Tinassi.

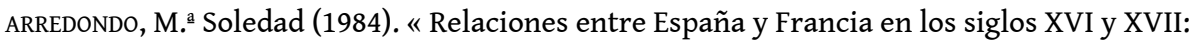
testimonios de una enemistad ». Dicenda. Cuadernos de filología hispánica, 3, 199-206.

BAGGIONI, Daniel (1997). Langues et nations en Europe. Paris : Payot.

BOURLAND, Caroline B. (1933). « The Spanish Schoole-Master and the Polyglot Derivatives of Noel de Berlaimont's Vocabulare ». Revue hispanique, LXXXI, 283-318.

BRUÑA, Manuel (2000). «L'enseignement de la prononciation française aux Espagnols $\left(\mathrm{XVI}^{\mathrm{e}}\right.$ et XVII ${ }^{e}$ siècles) ». Jan De Clercq et alii (éd.). Grammaire et enseignement du français, 1500-1700. Leuven : Peeters, 61-96.

BRUÑA, Manuel (2010). «Pedro Pablo Billet es el autor del arte « Arte para aprender (...) la lengua francesa (1672) ». J. C. De Miguel et al. (coord.). Enfoques de teoría, traducción y didáctica de la lengua francesa: Estudios dedicados a la profesora Brigitte Lépinette. Valencia : Universitat de Valencia, 103-112.

CORCUERA, J. Fidel \& GASPAR, Antonio (1999). La lengua francesa en España en el siglo XVI: Estudio y edición del 'Vocabulario de los vocablos' de Jacques de Liaño (Alcalá de Henares, 1665). Zaragoza : PUZ. CORREAS, Gonzalo (1903) [1626]. Arte grande de la lengua Castellana compuesto en 1626 por el Maestro G.C. Publicado por primera vez por el Conde de la Viñaza. Madrid : s. é.

FERNÁNDEZ-ORDÓÑEZ, Inés (2004). « Alfonso X en la historia del español ». In Rafael CANO (coord.). Historia de la lengua española. Barcelona : Ariel, 381-422.

FISCHER, Denise, GARCíA BASCUÑANA, Juan Francisco \& GóMEZ, Maria Trinidad (2004). Repertorio de gramáticas y manuales para la enseñanza del francés en España (1565-1940). Barcelona : PPU.

GASPAR, Antonio et CORCUERA, J. Fidel (2015). La gramática francesa de Baltasar de Sotomayor.

Zaragoza : PUZ. 
GONZÁLEZ OLLÉ, Fernando (1997). « Actitudes y actuaciones de Carlos V respecto a la lengua española ». In M. Almeida et J. Dorta (éds), Contribuciones al estudioinguís de la lingüísticainguística Hispánica, Santa Cruz de Tenerife : Montesinos, t. II, 309-332.

KAMEN, Henry A. (1997). Felipe De España. Madrid : Siglo XXI.

KUKENHEIM, L. (1932). Contributions à l'histoire de la grammaire italienne, espagnole et française à l'époque de la Renaissance. Amsterdam : Noord-hollandsche uitgevers-maatschappij.

LANOYE, Diederik (2002). « Benito Arias Montano and the University of Louvain (1568-1576) ». Lias, $29,23-44$

LÉPINETTE, Brigitte (1990). « La lexicographie franco-espagnole avant le Tesoro de las dos lenguas de César Oudin (1607) ». Travaux de linguistique et de philologie, XXVIII, 317-342.

LÉPINETTE, Brigitte (1996). « Les premières grammaires du français (1565-1799) publiées en Espagne. Modèles, sources et rôle de l'espagnol ». Histoire, Épistémologie, Langage, 18/II, 149-177.

LOPE BLANCH, Juan M. (1986). « La lingüística española del Siglo de Oro ». In Actas del Octavo Congreso de la Asociación Internacional de Hispanistas. Madrid : Istmo, 37-58. In :

http://www.cervantesvirtual.com/nd/ark:/59851/bmc7m276

MALDONAdo y PARDo, J. (1677). Museo o Biblioteca Selecta del Exc. Señor Don Pedro de Guzman, marqués de Montealegre. Madrid : Julián Paredes.

MARTíNEZ GAVILÁN, Mª Dolores (2002). « La Grammaire espagnole (1624) de Fray Diego de la Encarnación, un enfoque racionalista en la enseñanza del español como lengua extranjera ». In M. A. Esparza Torres, B. Fernández Salgado \& H.-J. Niederehe (éds.), Estudios de Historiografía Lingüística. Actas del III Congreso Internacional de la Sociedad Española de Historiografía Lingüística. Hamburg : Helmut Buske Verlag, 341-360.

MARTÍNEZ MILLÁN, J. \& FERNÁNDEZ CONTI, Santiago (dir.) (2005). La Monarquía de Felipe II: la Casa del Rey. Madrid : Mapfre.

MOREL-FATIO, Alfred (1901). Ambrosio de Salazar et l'étude de l'espagnol en France sous Louis XIII. Paris : Picard et fils.

NEBRIJA, Antonio de (1492). Gramática sobre la lengua castellana. Salamanca. http://bdh-rd.bne.es/ viewer.vm?id=0000174208\&page $=1$

NIEDEREHE, Hans J. (1994). Bibliografía cronológica de la lingüística, la gramática y la lexicografía. Desde los comienzos hasta el año 1600. Amsterdam : J. Benjamins.

oUdiN, César (1619) [1597]. Grammaire espagnolle expliquée en Francois. Bruxelles : Antoine. PALAU I DULCET, Antonio (1990). Manual del librero hispanoamericano : bibliografía general española e hispanoamericana desde la invención de la imprenta hasta nuestros tiempos. Madrid : Ollero. 7 vol. PEETERS-FONTAINAS, Jean-Félix (1965). Bibliographie des impressions espagnoles des Pays-Bas Méridionaux. Mise au point avec la collaboration de Anne-Mari Frédéric. Nieuwkoop : Graaf. PETTEGREE, A. Y WALSBY, M. (2011). Netherlandish books : books published in the Low Countries and Dutch books printed abroad before 1601. Leiden : Brill.

Recueil de Lettres missives de Henri IV (1858) t. VII, période 1606-1610. Paris : Imprimerie Impériale. SALAZAR, Ambrosio de (1623), Espejo general de Gramatica en dialogos para saber perfectamente la lengua Castellana con algunas historias muy graciosas y de notar. Rouen : Morron, 1623 [éd. fac. Slatkine Reprints]. 
SUÁREZ GÓMEZ, Gonzalo (2008) [thèse de 1956]. La enseñanza del francés en España hasta 1850. ¿Con qué libros aprendían francés los españoles ? éd. J. F. GARCíA BASCUÑANA y E. JUAN. Barcelona : PPU.

VACA DE OSMA, José Antonio (1999). Carlos I y Felipe II frente a frente. Madrid : Rialp.

VILLALón, Cristóbal (1997). El Scholástico. (Éd.) Martínez Torrejón. Barcelona : Crítica.

VILLALón, Cristóbal (1558). Gramatica Castellana: Arte breve y compendiosa para saber hablar y escrevir en la lengua castellana. Anvers : G. Simon.

VIÑAZA, Conde de la (MUÑoz y MANZANo, C.) (1893). Biblioteca histórica de la filología castellana.

Madrid : Real Academia Española.

YLLERA, Alicia (1983). « La gramática racional castellana en el siglo XVII, la herencia del brocense en España » In Serta philologica : F. Lázaro Carreter : natalem diem sexagesimum celebranti dicata. Vol. $1,649-666$.

YLLERA, Alicia (2016). « Deux grammairiens du XVII ${ }^{\mathrm{e}}$ siècle en Espagne et leur enseignement de la prononciation française : Pedro Pablo Billet et Juan Pedro Jaron ». In Antonio Gaspar et J. Vicente (éds.). Histoire de l'enseignement de la prononciation du français aux Espagnols (XVI ${ }^{e}-\mathrm{XX}^{e}$ siècles). Bern : Peter Lang, 43-62.

\section{NOTES}

1.

Baggioni (1997 : 94) reconnaît l'existence d'une langue commune préalable dans le processus de conformation d'une langue nationale: «Dans les cas des langues nationales en Europe, [...] la distinction d'une phase langue commune antérieure à celle de langue nationale est pertinente ». La diversité linguistique à l'intérieur des territoires français et espagnol est pourtant riche. Salazar (1623 : 49) écrit : « Sçachez qu'en Espagne c'est ny plus ny moins qu'en France, comme il y a diverses Provinces, aussi il y a diverses langues ».

2. En 1932, Kukenheim (1932: 198) se faisait l'écho du patriotisme des grammaires de la Renaissance : «À la lecture des grammaires parues au cours du XVI ${ }^{\mathrm{e}}$ siècle, on est frappé du ton d'ardent patriotisme qui se fait entendre là où l'auteur s'explique sur le but de son travail ».

3. À partir de 1241, la plupart des documents de la Chancellerie de Castille et Léon sont écrits en castillan. (Fernández-Ordóñez, $2004: 394$ ).

4. Le vocabulaire plurilingue écrit par Ambrosio Calepino, a été l'un des manuels des langues les plus publiés à partir de 1550. L'édition de Lyon de 1559 compte une nouvelle langue, la langue castillane, d'où son importance dans le contexte international. À partir de cette édition, l'ouvrage comptera d'autres langues, dont le français, l'allemand, le flamand...), et sera publié dans les principales villes européennes. Pourtant, ce dictionnaire n'a jamais été publié en Espagne.

5. Recueil de Lettres missives de Henri IV, t. VII, 1606-1610, Paris, Imprimerie Impériale, 1858, p. 665.

6. J. F. Peeters-Fontainas (1965) fait un rapport des œuvres espagnoles publiées aux Pays Bas et affirme qu'il existe mille quatre cent treize impressions d'œuvres des grands auteurs espagnols de l'époque entre 1520 et 1785.

7. Pour le rôle international de l'espagnol à l'époque, voir González Ollé (1997).

8. Pour une perspective générale de l'activité linguistique des Espagnols pendant le Siècle d'Or, consulter Lope Blanch (1986).

9. Pour les détails des œuvres voir D. Fischer, J. F. García Bascuñana et Mª T. Gómez (2004) et Gonzalo Suárez (2008).

10. La date de la licence est du 30 mai 1564. La Grammaire a été publiée en un seul volume avec le Vocabulaire de Liaño, par les mêmes imprimeurs à Alcalá de Henares, ce qui explique la date de 
publication. Il existe une édition critique moderne précédée d'une étude sur l'auteur, les exemplaires et le contenu de l'ouvrage rédigée sous les soins de Gaspar et Corcuera (2015).

11. Jacques Ledel -o Liaño- arrive en Espagne en 1560 dans le cortège de la reine Isabelle de Valois, épouse de Philippe II. Pour toutes les questions concernant Ledel et son Vocabulario, on peut consulter l'édition critique réalisée par Corcuera et Gaspar (1999) et publiée par les Presses de l'Université de Zaragoza.

12. L'auteur signale dans la dédicace « al curioso lector español », que cette grammaire a été déjà publiée une première fois à "Douay, Universidad del Condado de Flandes, año M. DC. XXIV », avec un nom d'auteur différent, Fray Diego de la Encarnación, son nom religieux de «carme déchaussé ».

13. En réalité il ne s'agit pas d'un dictionnaire, mais d'un mélange de vocabulaire, conseils de prononciation et modèles de dialogues suivant la série des Colloques de Berlaimont.

14. Il s'agit d'une copie des œuvres de Liaño et Sotomayor de 1565, adaptée aux goûts du marché, qui présente des changements considérables: réduction du nombre de pages (de 232 à 142), disparition du prologue, des noms des auteurs et d'une bonne partie du Vocabulaire de Liaño.

15. Billet est aussi l'auteur de la traduction en espagnol d'un livre de mémoires intitulé La verdad en su luz o Las verdaderas memorias de madama Maria de Machini, Condestable Colona. (Zaragoza, 1677). 16. Billet déclare qu'il a été tellement déçu par le résultat, qu'il a rédigé une nouvelle édition. Certains auteurs (Bruña, 2010 : 79; Yllera, 2016 : 48) considèrent que cette édition antérieure est l'Arte para aprender facilmente a leer, escrivir y hablar la Lengua Francesa, por Don P. B. Maestro de Lenguas. En Leon de Francia, a costa de Pedro Burgea, Mercader de Libros en Madrid, paru en 1672. L'impression a été payée par un marchand de Madrid, ce qui laisse supposer que la publication à Lyon est une question purement économique ou d'opportunité, et que la grammaire était destinée au marché espagnol. Palau (1999 : Réf. 32505) attribue cet ouvrage à Pierre Bonet et Maldonado y Pardo (Madrid, 1677 : 24) écrit : « Arte para aprender facilmente a leer, escrivir y hablar la Lengua Francesa, por Don Pedro Bonet, Maestro de las lenguas, en Leon, año de 1672 ». Cette référence de 1677 est peut-être à l'origine de l'attribution de Palau.

17. L'édition de 1688 contient une annexe de 16 pages -ajoutée à posteriori à la Grammaireintitulée « Dissertacion Critica, sobre una Cuartilla, que con nombre de Arte, saco a luz el Señor Juan Pedro Jaron ", conservée malheureusement de façon incomplète à la Bibliothèque Nationale de Madrid (Réf. 3/57521) et qui adresse des critiques virulentes contre Jaron.

18. Le titre du manuscrit est le suivant: Arte Grammatica y manera de bien hablar, screbir y leer la lengua Francesa breue y conpendiosa para los que sauen Romançe/nueuamente compuesta por el Maestro Balthasar Pérez del Castillo, Canónigo de la Sancta Iglesia y natural de Burgos y Rector de Fuenlabrada (Exemplaire II/1765 et microfilm MC/1499). Cet opuscule inédit a été trouvé par L. Pablo Nuñez (2011) qui en a publié une étude et une édition critique dans le Bulletin de la Real Academia Española.

19. Pour toutes les questions concernant Sotomayor et sa grammaire, on peut consulter notre travail (Gaspar et Corcuera, 2015) qui comprend une étude de l'auteur, une analyse des sources et des exemplaires conservés de la Grammatica, ainsi qu'une édition critique. 


\section{ABSTRACTS}

The Spain of the imperial era is a linguistic mosaic. Several Romance languages, such as the Spanish, Catalan, Aragonese or Galician, coexist with the Basque and Arabic. The incorporation of the "New World" into the Crown increases institutional linguistic concerns. From 1492, it's necessary to learn the languages of Native Americans for military, religious or economic reasons. In the European context, the Spanish empire and France are two rival powers competing for world supremacy. In this scenario so unpropitious for cultural exchange, the French textbooks published in Spain are generally exceptional individual adventures. Our contribution reflects this historical period and analyses the books and authors who have pioneered the teaching of French in Spain.

L'Espagne de l'époque impériale est une mosaïque linguistique: plusieurs langues d'origine latine, dont le castillan, le catalan, l'aragonais ou le galicien, coexistent avec le basque et l'arabe. L'incorporation du « Nouveau Monde » à la couronne augmente les préoccupations linguistiques institutionnelles. À partir de 1492, il faut apprendre les langues des peuples amérindiens pour des raisons militaires, religieuses ou économiques. Dans le contexte européen, l'empire espagnol et la France sont deux puissances rivales qui se disputent la suprématie mondiale. Dans cette ambiance si peu propice aux échanges culturels, les manuels de français publiés en Espagne constituent, en général, des aventures individuelles exceptionnelles. Notre contribution rend compte de cette période historique et analyse les œuvres et les auteurs qui ont été pionniers de l'enseignement du français en Espagne.

\section{INDEX}

Keywords: Spanish Empire, Language policy, French textbook, FLE

Mots-clés: Espagne impériale, politique linguistique, manuels de français, FLE

\section{AUTHORS}

\section{ANTONIO GASPAR GALÁN}

Université de Zaragoza

agaspar@unizar.es

\section{J. FIDEL CORCUERA MANSO}

Université de Zaragoza

corcuera@unizar.es 